\title{
Hybrid flex foil-ferrite technology for miniaturized power and RF applications
}

\author{
M. Saidani and M.A.M. Gijs \\ Institute of Microelectronics and Microsystems, Swiss Federal Institute of Technology Lausanne, \\ CH-1015 Lausanne EPFL, Switzerland. E-mail: menouer.saidani@epfl.ch and martin.gijs@epfl.ch
}

\begin{abstract}
A hybrid technology for the realization of threedimensional miniaturized power inductors, transformers and RF inductors is presented. This technology consists of combining planar $\mathrm{Cu}$ coils on polyimide substrates with high permeability and high resistivity ferrite cores. For miniaturized power inductors and transformers, high permeability $\left(\mu_{r} \sim 2000\right) \mathrm{Mn}-\mathrm{Zn}$ ferrite cores are used. These cores operate up to $10 \mathrm{MHz}$ and are obtained by threedimensional micro-patterning of ferrite wafers using the powder-blasting technique. High resistivity $\left(10^{7} \Omega \mathrm{cm}\right) \mathrm{Ni}-\mathrm{Zn}$ ferrite plates cores are used for the realization of RF inductors. The plates are deposited on top of the coils to enhance the magnetic properties of the inductor at $\mathrm{GHz}$ frequencies. The planar $\mathrm{Cu}$ coils are realized using an inhouse developed high-resolution polyimide spinning and $\mathrm{Cu}$ electroplating process on a flexible support of Kapton. Inductive and resistive properties of the power inductors are characterized as a function of frequency; inductance values in the $1 \mathrm{mH}$ range have been obtained. RF inductors show a 40 $\%$ enhancement of the inductance and a $25 \%$ enhancement of the quality factor for frequencies up to $0.2 \mathrm{GHz}$. Our results indicate the potential of using bulk ferrites in a hybrid assembly process for power and RF applications.
\end{abstract}

Index Terms - Inductor, flex-foil, ferrite, powder blasting, RF inductor

\section{INTRODUCTION}

Ferrite materials provide interesting options for miniaturized power and radio frequency (RF) applications. This is due to two reasons: first, their high relative permeability factors $\left(\mu_{r} \sim 10^{2}-10^{3}\right.$ ) allow obtaining high inductance values with minimal number of turns for power or transformation applications; secondly, their high resistivity ( $\rho \sim 10-10^{7} \Omega \mathrm{cm}$ ) reduces eddy current losses enabling RF applications at $\mathrm{GHz}$ frequencies.

Bulk ferrite materials may present an interesting alternative option for use as a magnetic material instead of metallic magnetic materials. Sputtering [1], spin coating [2] or plating [3] techniques have been proposed for the realization of ferrite films, but such films often require a post-deposition annealing up to $1000{ }^{\circ} \mathrm{C}$, which most of the time is not compatible with Integrated Circuit (IC) postprocessing. Pulsed laser deposition of high-quality (MnZn)Fe ferrite films, showing bulk magnetic properties, was achieved at $400-600{ }^{\circ} \mathrm{C}$ by a careful selection of buffer layers [4]. However, there appears to be no readily available technique for fabrication of ferrite films with the desired magnetic properties at a sufficiently low temperature that is compatible with an IC-based process.

\section{HIGH-RESOLUTION FLEXIBLE COILS}

The present work comprises the microfabrication of multilayered $\mathrm{Cu}$ coils on a flexible polyimide (Kapton) substrate. These coils have the advantage of being detachable from the Si substrate, are made in a suitable IC post-processing technology, and can be combined with other substrates, in particular with either structured (Ecores) or non-structured (plates) ferrites. The coil microfabrication involves various lithography, polyimide spinning, $\mathrm{Cu}$ electroplating, planarization and plasma etching steps. This Si wafer-based process has the potential of realizing a minimum winding width $w=5 \mu \mathrm{m}$ and a minimum winding pitch $p=15 \mu \mathrm{m}$. Figure 1 shows the fabrication sequence of these high-resolution coils (for more details see [5]).

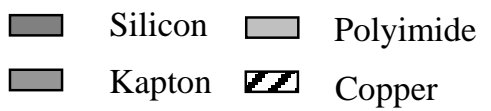

(a)

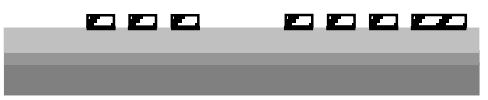

(b)

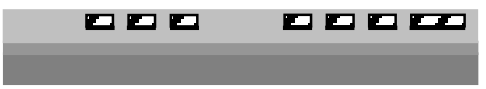

(c)

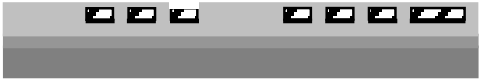

(d)

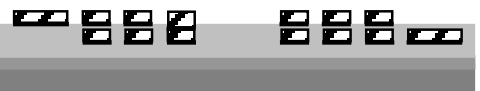

(e)

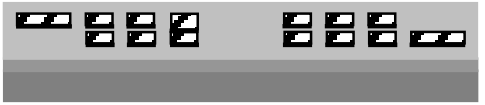

(f)

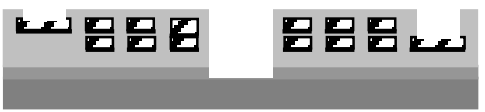

(g)

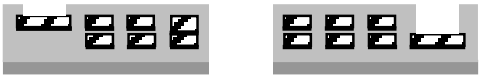

Fig. 1, Fabrication sequence for high-resolution polyimide-based double layered coils on a flexible Kapton support: (a) electroplating of the first layer of the coil, (b) polyimide isolation layer deposition, (c) via hole etching, (d) electroplating of the second layer of the coil, (e) polyimide isolation layer deposition, (f) feed-through hole and contact path holes etching (g) final separation of the flexible structures from the Si substrate 
For each plasma etching step, an $\mathrm{SiO}_{2}$ mask is PECVDdeposited and lithographically structured, after which the polyimide is etched in a $\mathrm{O}_{2}$ plasma to create the throughholes for the positioning of the E-cores (for the power inductors) and to clear the contact paths (Fig. 1(f)). To separate the flexible structures from the Si substrate, a bath of acetone is used to remove the adhesive between the Kapton film and the wafer (Fig. 1(g)).

\section{POWER FERRITE INDUCTORS}

For the magnetic power ferrite cores, we use high relative permeability $\left(\mu_{\mathrm{r}} \sim 2000\right)$ 3F3 ferrite wafers from Ferroxcube [6]. Three types of three-dimensional ferrite Ecores are micromachined in $1 \mathrm{~mm}, 0.75 \mathrm{~mm}$ and $0.5 \mathrm{~mm}$ thick ferrite wafers using mechanical micro-erosion by powder blasting [7].

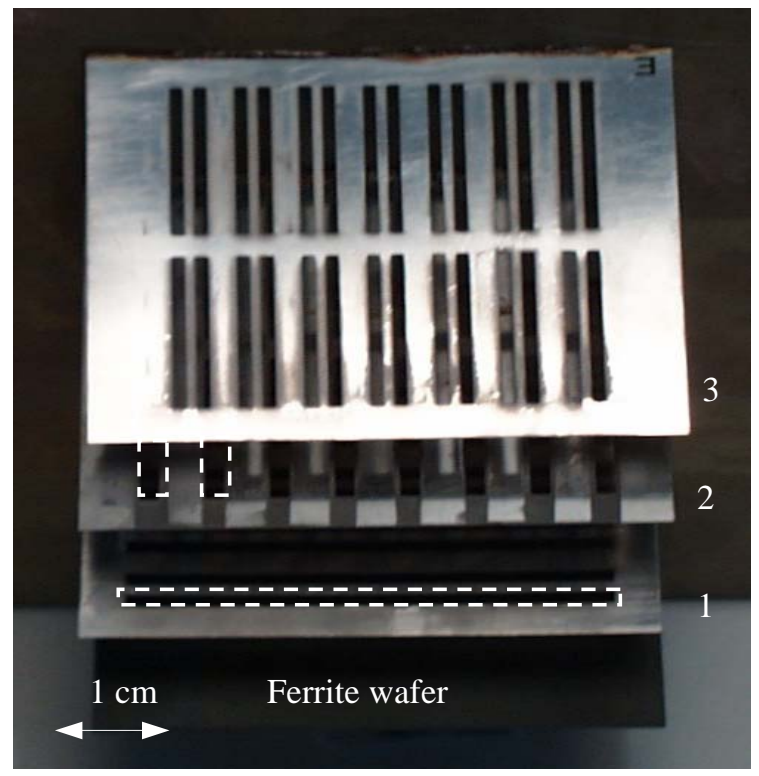

Fig. 2, Successively used powder blasting masks.

Three $0.5 \mathrm{~mm}$ thick stainless steel masks, shown in figure 2, are subsequently used to realize an array of ferrite E-cores. During the powder blasting process, these masks are temporarily glued to the ferrite wafer to promote the mechanical contact and to prevent underetching effects. By performing an $\mathrm{x}$ and $\mathrm{y}$-axis translation of the powder blasting nozzle, we obtain a uniform exposure of the fixed ferrite wafer. Figure 3(a) is a SEM photograph of the smallest 3D E-core we realized with our technique. Figure 3(b) is a photograph of an assembled $10 \mathrm{~mm}^{3}$ size transformer by combination of two cores with a flex foil coil.

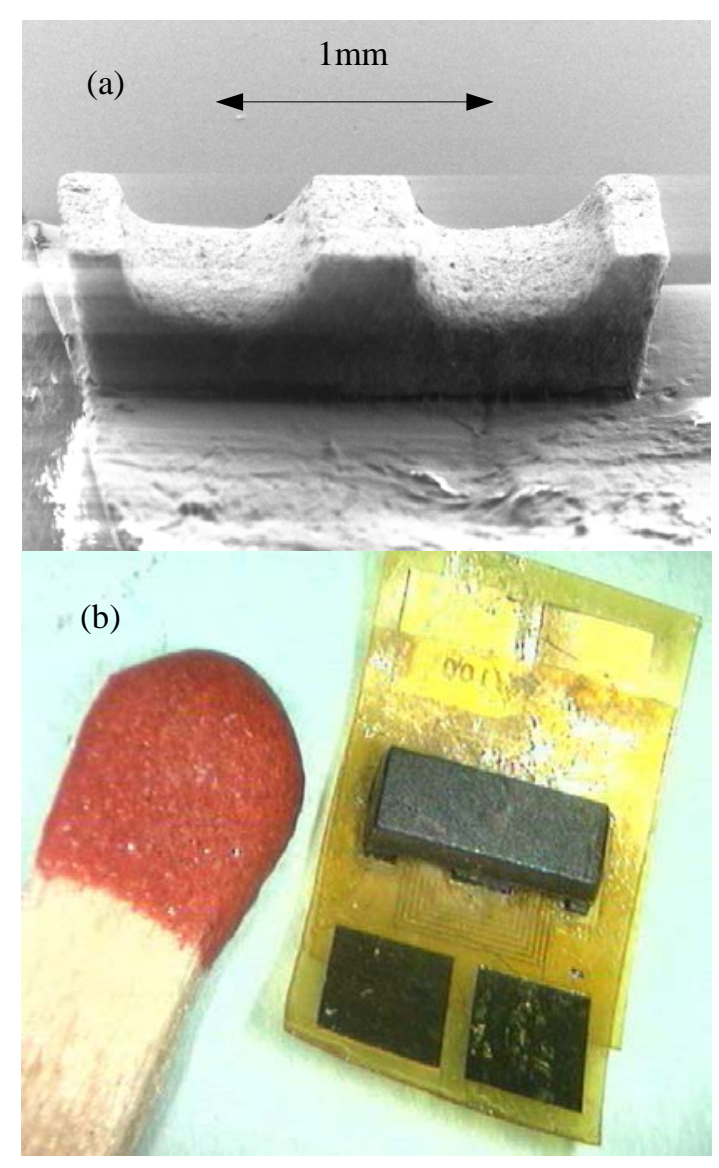

Fig. 3, (a) SEM photograph of the smallest microstructured single ferrite E-core (2 mm x $0.71 \mathrm{~mm}$ x $0.5 \mathrm{~mm}$ ); (b) a final assembled inductor

The flexible coils and the E-cores are assembled, whereby the two E-cores auto-align as a consequence of minimization of magnetic demagnetization energy. The Ecores are then glued and mechanically clamped together to reduce air gap losses.

Figure 4(a) and figure 4(b) show inductance and quality factor measurements, respectively, of an assembled inductor with and without the application of the two ferrite E-cores respectively. One clearly sees the strong enhancement of the inductance in the $\mathrm{mH}$ range $\left(L_{\text {avg }} \sim 0.6\right.$ $\mathrm{mH}$ ) over four decades of frequencies due to the presence of the ferrite and corresponding to a 50 times enhancement with respect to the inductance value without using a ferrite core. Also the quality factor is enhanced and its value can be easily further enhanced by a factor of 2 or 3 by thickening the metal in the coil. 
(a)

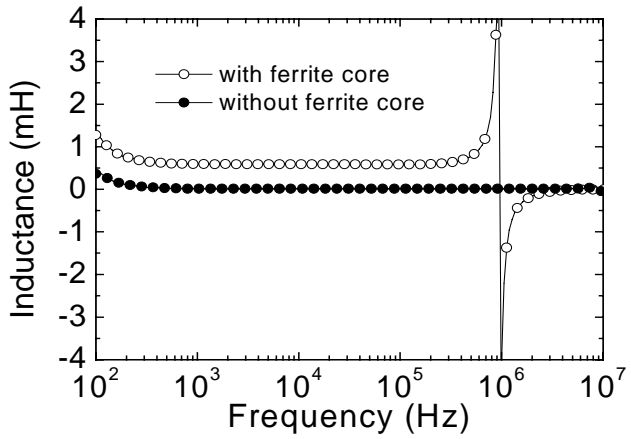

(b)

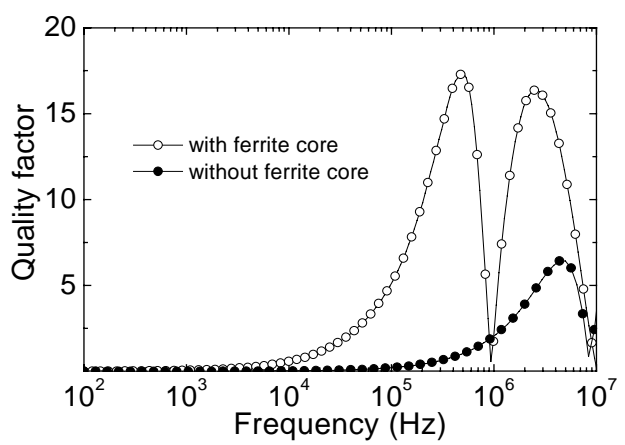

Fig. 4, Dependence of the inductance (a) and the quality factor (b) on frequency for a coil with a number of windings $N=58$ with and without two ferrite cores of size $6 \times 2 \times 1 \mathrm{~mm}^{3}$ each

\section{RF INDUCTORS}

For the realization of RF inductors, the polyimide based $\mathrm{Cu}$ coils fabricated on top of normal Si substrates ( $\rho$ $\sim 0.1-1 \Omega \mathrm{cm}$ ) are loosely assembled with magnetic cover plates of commercially available bulk Ni-Zn ferrites of high resistivity (see table I).

\section{$d$}

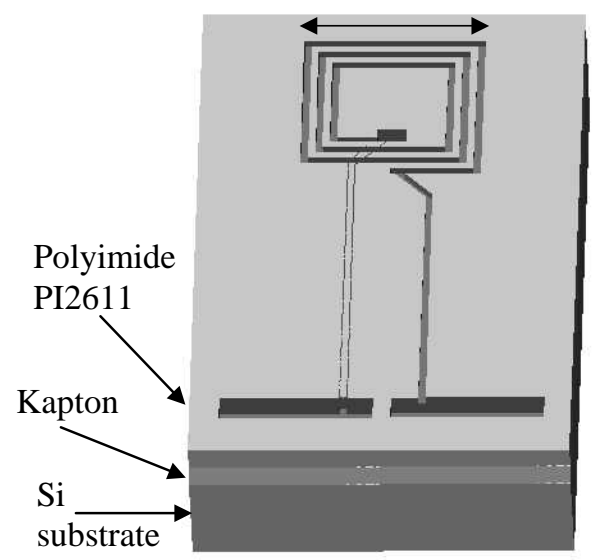

Fig. 5, Oblique schematic view of a $\mathrm{Cu}$ coil of diameter $d$ encapsulated in a polyimide PI2611 structure, which is realized on top of a Kapton film that is detachable from the Si substrate

$\mathrm{Ni}$-Zn ferrites are generally used for electromagnetic interference suppression applications and have an appreciable magnetic permeability up to $1 \mathrm{GHz}$, the highest frequency with still a magnetic behavior among the ferrites with cubic anisotropy [8]. Their very high resistivity prevents eddy currents losses, present in metallic magnetic materials, and only magnetic resonance effects can cause dissipation at $\mathrm{GHz}$ frequencies.

Following a similar process to that of figure 1, RF coils have been fabricated, the design of which is shown in figure 5 . The coil has a size $d$ in the range $0.5 \mathrm{~mm}<d<2 \mathrm{~mm}$ and consists of $15 \mu \mathrm{m}$ thick and $40 \mu \mathrm{m}$ wide $\mathrm{Cu}$ windings ( $\mathrm{N}=1-3)$ with a winding pitch of $80 \mu \mathrm{m}$. A schematic crosssection of the coil is shown in Fig. 6(a). The embedded coil can be simply covered with a ferrite plate (Fig. 6(b)) or is easily detachable from the silicon substrate (Fig. 6(c)). We have used $1 \mathrm{~mm}$ thick and $1 \mathrm{~cm}$ wide square plates of four different Ni-Zn ferrites listed in Table I, all having a resistivity of the order of $10^{7} \Omega \mathrm{cm}$. Alternatively, such ferrite plates can be subdivided by micromachining and combined with the coils in a batch-type gluing or assembly process.

Table I, Magnetic permeability at $1 \mathrm{MHz}$ of the selected ferrite materials, as specified by the manufacturer

\begin{tabular}{|c|c|}
\hline $\begin{array}{c}\text { Ferrite grade } \\
\text { (Ferroxcube) }\end{array}$ & $\begin{array}{l}\text { Initial permeability } \\
\text { @ 1 MHz }( \pm 20 \%)\end{array}$ \\
\hline 4C65 & 125 \\
\hline 4E1 & 15 \\
\hline 4E2 & 25 \\
\hline 4F1 & 80 \\
\hline
\end{tabular}

We have measured the inductance, resistance and quality factor of the inductors in the $0.001-3 \mathrm{GHz}$ range using an Agilent E4991A RF Impedance/Material Analyzer and a Cascade Microtechnique probe station. We present in this paper results for coils in the three configurations of figure 6. Measurements are done first on the silicon substrate with only the $\mathrm{Cu}$ coil on top (fig. 6(a)); then a ferrite cover plate is deposited on top of the coil (fig. 6(b)), and finally the inductor with ferrite cover is removed from the Si substrate (fig. 6(c)). Results for the three configurations are shown in figure 7.

Figure 7(a) is the frequency-dependent inductance for two coils with an outer diameter $d=750 \mu \mathrm{m}$ and a number of turns $\mathrm{N}=1$ and $\mathrm{N}=3$, respectively. When $\mathrm{N}=1$, we clearly observe the enhancement of the inductance due to the presence of the ferrites, irrespective from the presence of the $\mathrm{Si}$ substrate. For $\mathrm{N}=3$, we observe a more distinct resonant-like behavior: the inductance tends to increase when approaching the resonance frequency $f_{0}=2.5 \mathrm{GHz}$ $\sim 1 / 2 \pi \sqrt{L C_{p}}$, from which we derive an effective parasitic capacitance $\mathrm{C}_{\mathrm{p}}=0.22 \mathrm{pF}$. The general observation from figure $7(\mathrm{a})$ is the pronounced increase of the inductance (of the order of $40 \%$ ) due to the presence of the ferrite and the strong similarity for the different ferrites used. The reduced inductance values when both the ferrite and the Si substrate are present can be simply explained by eddy current contributions from the $\mathrm{Si}$, which have a 
screening effect and tend to decrease the magnetic flux through the coil.

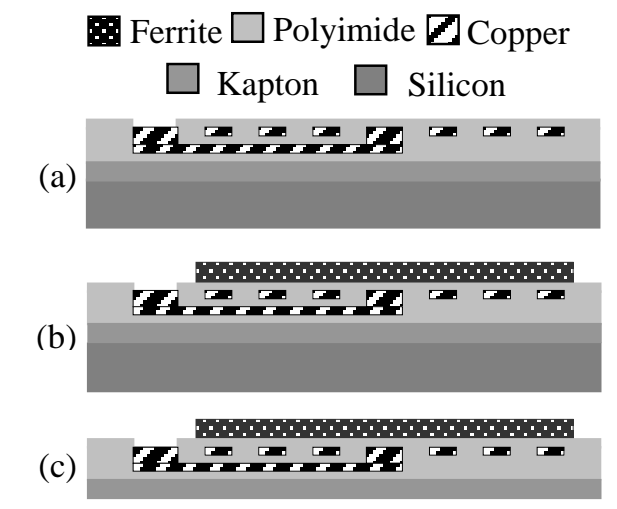

Fig. 6, Illustration of the chosen configurations: (a) polyimide embedded coil on top of the Si substrate; (b) coil on the Si substrate with a ferrite cover plate; (c) coil with ferrite cover plate and detached from the Si substrate

(a)

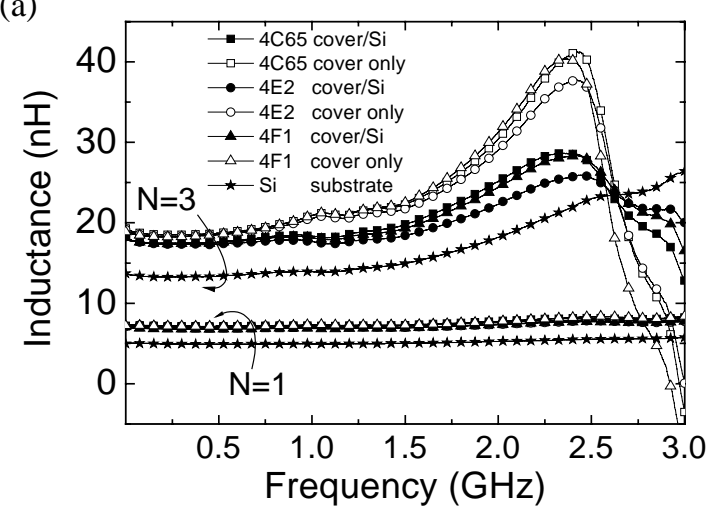

(b)

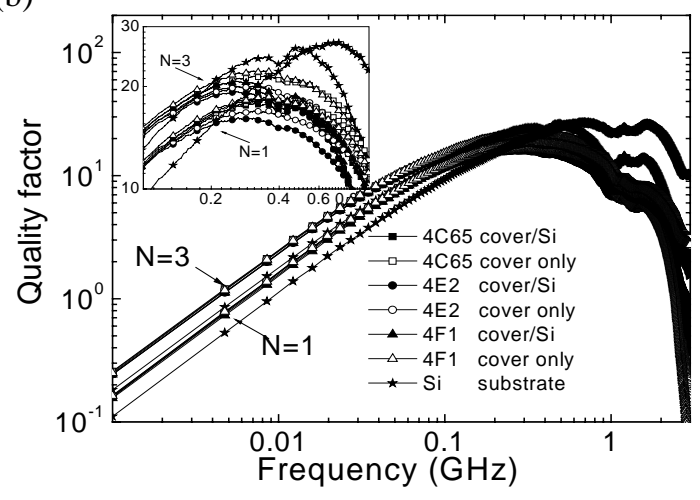

Fig. 7, Frequency dependence of (a) the inductance and (b) the quality factor for the experimental configurations of figure 6 (using three different ferrites). The coils have an external size $d=0.75 \mathrm{~mm}$ and a number of windings $\mathrm{N}=1$ and $\mathrm{N}=3$, respectively. The insert of figure $7(b)$ is a zoom on the quality factor behaviour at high frequencies

Figure 7(b) shows the quality factor $Q=\omega L / R$ of the same set of samples. The strongly increased inductance results in a quality factor enhancement of $25 \%$ with respect to the simple coil, up to a frequency of $0.2 \mathrm{GHz}$. At higher frequencies, the ferromagnetic resonance losses of the ferrite decrease the quality factor to a value below that of the device with Si substrate only.

The inset of figure 7 (b) more clearly shows the behavior of the highest Q values: at around $0.3 \mathrm{GHz}$, there is a crossing of the family of curves with ferrite cover plate only, and the curves for the coils with both the Si substrate and ferrite plate present. Herewith we demonstrate the limiting frequency for the use of high-resistivity cubic ferrites for RF inductor applications.

\section{CONCLUSION}

By combining powder blasting microstructured plates of commercially available $\mathrm{Mn}-\mathrm{Zn}$ ferrites with flex foil processed coils, we have realized miniaturized threedimensional (3D) batch-type inductors for power applications (0.1-1 W). This method makes use of the excellent magnetic properties of the bulk material resulting in higher inductances and quality factors compared to metallic and ferrite thin film-type magnetic materials. For $\mathrm{RF}$ applications in the $\mathrm{GHz}$ range, we obtain a strong enhancement of the inductance and quality factor using $\mathrm{Ni}$ $\mathrm{Zn}$ ferrites. Above $0.3 \mathrm{GHz}$, ferromagnetic resonance losses in the ferrite dominate. Further extension of the useful frequency range would rely on the development of new types of ferrites with increased ferromagnetic resonance frequency.

\section{REFERENCES}

[1] I. Zaquine, H. Benazizi, and J. C. Mage, "Ferrite thin-films for microwave applications”, J. Appl. Phys., vol. 64, no. 10, Part 2 Nov 1988, 5822-5824.

[2] J.Y. Park, L.K. Lagorce, and M.G. Allen, "Ferrite-based integrated planar inductors and transformers fabricated at low temperature”, IEEE Trans. Magn., vol. 33, no. 5, Part 1 Sep 1997, 3322-3324.

[3] M. Abe, "Oxide ferromagnetic films and fine particles prepared by ferrite plating and their applications”, Electrochemistry, vol. 70, no. 10, Oct 2002, 815-820.

[4] Y. Suzuki, R. B. van Dover, E. M. Gyorgy, J. M. Philips, V. Korenivski, D. J. Werder, C. H. Chen, R. J. Cava, J. J. Krajewski Jr., W. F. Peck, and K. B. Do, "Structure and magnetic properties of epitaxial spinel ferrite thin films”, Appl. Phys. Lett., vol. 68, no. 5, Jan 1996, 714-716.

[5] M. Saidani and M.A.M. Gijs, "Cubic millimeter power inductor fabricated in batch-type wafer technology”, J. Microelectromechanical Syst., vol. 12, no. 2, Apr 2003, 172-178.

[6] Soft Ferrite and Accessories Catalogue (2002) on http://www.ferroxcube.com

[7] E. Belloy, S. Thurre, E. Walckiers, A. Sayah and M. A. M. Gijs, "The introduction of powder blasting for sensor and microsystem applications”, Sensors and Actuators A, vol. 84, no. 3, Sep 2000, 330-337.

[8] S. Chikazumi and S.H. Charap, Physics of magnetism, John Wiley \& Sons, New York, 1964. 